Research Article

\title{
Controller Design of Complex System Based on Nonlinear Strength
}

\author{
Rongjun Mu and Chao Cheng \\ School of Astronautics, Harbin Institute of Technology, Harbin 150001, China \\ Correspondence should be addressed to Chao Cheng; chchengao@126.com
}

Received 21 February 2015; Accepted 3 May 2015

Academic Editor: Rafael Morales

Copyright ( 2015 R. Mu and C. Cheng. This is an open access article distributed under the Creative Commons Attribution License, which permits unrestricted use, distribution, and reproduction in any medium, provided the original work is properly cited.

\begin{abstract}
This paper presents a new idea of controller design for complex systems. The nonlinearity index method was first developed for error propagation of nonlinear system. The nonlinearity indices access the boundary between the strong and the weak nonlinearities of the system model. The algorithm of nonlinearity index according to engineering application is first proposed in this paper. Applying this method on nonlinear systems is an effective way to measure the nonlinear strength of dynamics model over the full flight envelope. The nonlinearity indices access the boundary between the strong and the weak nonlinearities of system model. According to the different nonlinear strength of dynamical model, the control system is designed. The simulation time of dynamical complex system is selected by the maximum value of dynamic nonlinearity indices. Take a missile as example; dynamical system and control characteristic of missile are simulated. The simulation results show that the method is correct and appropriate.
\end{abstract}

\section{Introduction}

In recent years, many countries have begun to develop and explore the dynamics and control problems of complex systems. In the field of aviation and aerospace, the dynamic complex system means that vehicles have the difficulties of strong mobility, wide flight envelope, large Mach number range, complex control mechanism, dramatic changes of aerodynamics parameters, numerous uncertain factors, and so forth [1]. At each stage of the mission, vehicles are affected by all sorts of uncertainties and disturbances. These factors make the flight dynamics present the obvious characters of strong nonlinearity and tight coupling. Therefore, the dynamics modelling, uncertainty analysis of dynamical model, and design of flight control system become the hot spot and difficult spot in this research area. The analysis of flight dynamics and control system design are an important guarantee of completing the assigned task securely and reliably.

Linear theory is always the top priority for researchers to analyze and design the flight dynamics and control system. However, the approximately linearized method of nonlinear system equations needs to be based on some acceptable tolerances. Because of the strong coupling and high nonlinear property of some vehicles, these assumptions and limited conditions might not be suitable. Therefore, the dynamics and controller as a complex nonlinear system are not simplified as a linear model. The nonlinear dynamics can be analyzed through more complicated methods such as Volterra series and bifurcation [2]. The complex control system can be designed by the robust adaptive control strategy or more advanced control algorithm [3]. Before using these techniques, the efficiency, calculation complexity, and approximation precision of the method should be comprehensively considered. This raises a significant question: what is the nonlinear degree of the dynamical model? This question needs to be asked qualitatively and quantitatively. However, before considering this question, a more important point is that we must find a method to measure the nonlinear strength of dynamical model.

The nonlinearity index method emerged as the times required. Junkins and Singla first proposed nonlinearity index method, which is applied to measure the nonlinear strength of orbit and attitude dynamics [4]. In this paper, a series of simulation results show that the nonlinearity index 
can provide a feasible method to assess the system's nonlinearity. These simulation examples are also proposed relative to initial value problems and coordinate selection. Omran and Newman applied the nonlinearity index theory to aircraft flight mechanics analysis and proposed four expressions [5]. The four expressions are static state, static input, dynamic state, and dynamic input nonlinearity indices, which indicate the effect of input excitation and parameterized models on the nonlinear strength of aircraft flight mechanics.

Bringing the nonlinearity index to gain scheduling method is a totally new idea to design controller. Unlike the traditional design method of missile controller, the new design idea does not need to choose feature points on the trajectory of missile for designing the controller, just considering the entire flight envelope with respect to Mach number and angle of attack. The nonlinearity index provides a method to systematically quantify the nonlinearity strength scale over the entire flight envelope. According to the strong and weak nonlinearity, dividing the flight envelope into various phases, in each phase, different controllers are designed.

The paper is outlined as follows: Section 2 briefly describes the static and dynamic nonlinearity index theory, and the theory is extended to parameterized full flight envelope settings of the missile. In Section 3, based on nonlinearity index theory, the control system is designed. Based on the different nonlinear strength, the full flight envelope is divided into four parts. Section 4 starts by applying nonlinearity indices to a missile model. Then the characteristic of the missile control system is analyzed. Finally, conclusions are drawn in Section 5.

\section{A Measurement Method of Nonlinear Strength}

2.1. Nonlinear Measurement for Algebraic Systems. Consider an algebraic system

$$
y=f(x)
$$

where $f(x)$ is a differentiable function, $x \in R^{n}, y \in R^{m}$.

Now a reference state $\bar{x}$ is defined; then the mapping result is given through $y=f(x)$ as

$$
\bar{y}=f(\bar{x}) .
$$

In certain vicinity of the reference state $\bar{x}$, there is a family of finite $x$-variations: $x=\bar{x}+\delta x$. The $x$-variations map through (1) into the corresponding finite $y$-variations as

$$
\begin{aligned}
y & =f(\bar{x}+\delta x), \\
\|\delta x\| & \leq \delta x_{\text {max }} .
\end{aligned}
$$

However, this mapping may be linear or nonlinear over the region. Figure 1 shows an example of a nonlinear mapping for $m=2$ and $n=2$ case. The accuracy of linear approximation of the algebraic model is a positive way to analyze the nonlinear strength.

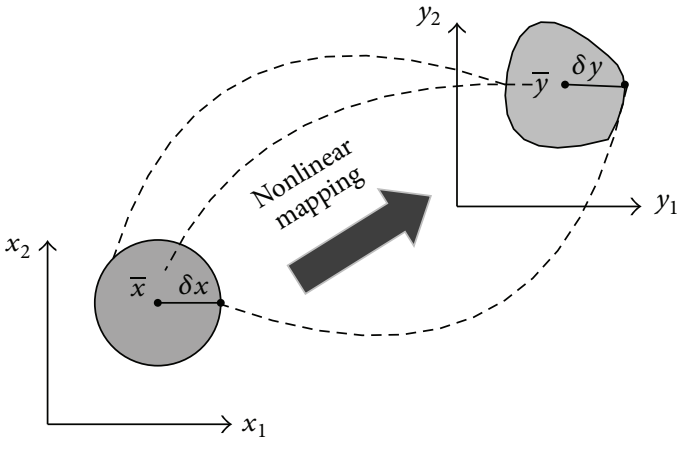

Figure 1: A nonlinear mapping.

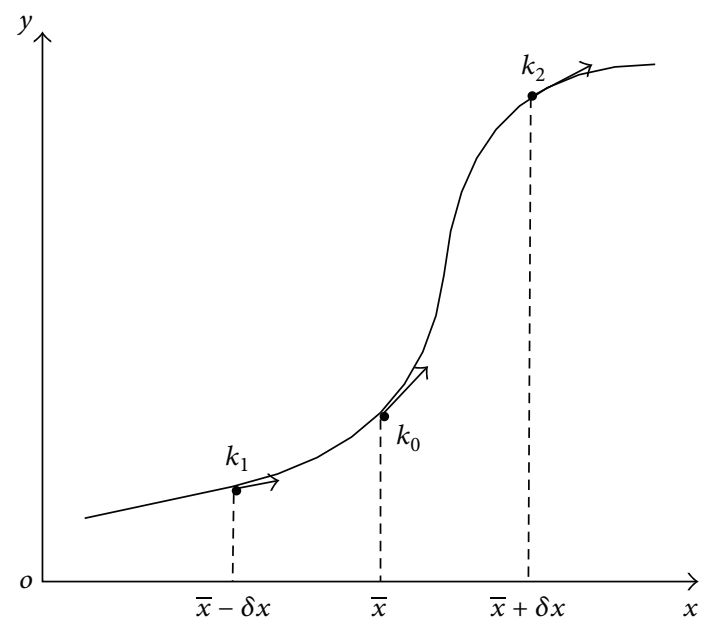

Figure 2: The curve $y=f(x)$.

In the bounded region, the linear approximation is computed as follows:

$$
\begin{aligned}
\delta y & =f(\bar{x}+\delta x)-f(\bar{x}) \cong \bar{A} \delta x, \\
A(x) & =\frac{\partial f(x)}{\partial x}, \\
\bar{A} & =A(\bar{x})=\left.\frac{\partial f(x)}{\partial x}\right|_{x=\bar{x}} .
\end{aligned}
$$

The deviation between Jacobian matrix $A(x)$ and the reference $A(\bar{x})$ is an effective way to measure the nonlinearity index over the sphere $\|\delta x\| \leq \delta x_{\max }$ :

$$
v=\sup \frac{\|A(x)-A(\bar{x})\|}{\|A(\bar{x})\|},
$$

where $\|\cdot\|$ indicates the Frobenius norm.

Equation (5) indicates the largest departure from the reference state found in the Jacobian matrix. For explaining the mean of nonlinearity index, specifically consider a case of $m=1$ and $n=1$. Figure 2 shows an example of the curve $y=f(x)$. The Jacobian matrix $A(x)$ indicates the slope of the curve on the point $x$. If the algebraic model is linear, the value of nonlinearity index is almost zero. The high nonlinearity index means the strong nonlinearity. 


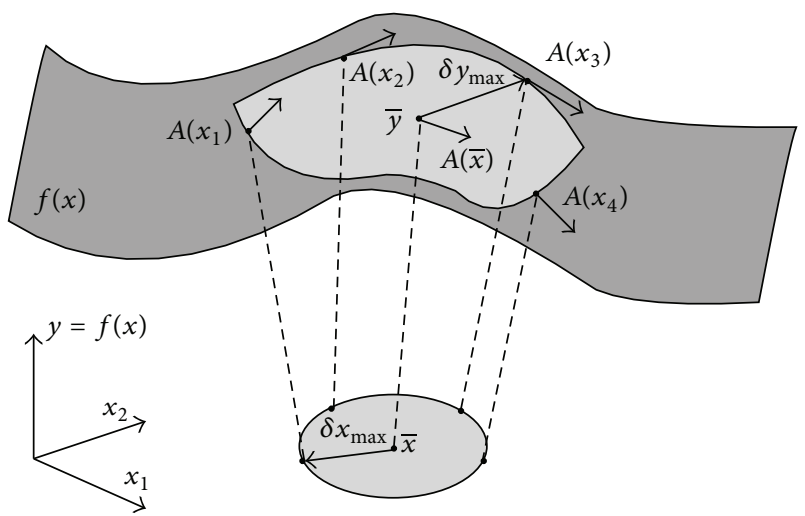

FIgURE 3: The idea of nonlinearity index of algebraic model.

In the algebraic model, the nonlinearity index indicates a deviation of the curvature in certain region. The static index evaluates the variations of Jacobian as a measure of nonlinearity. Figure 3 shows the idea of nonlinearity index of the algebraic model. Note that the value of nonlinearity index which is less than 0.01 indicates lower than $1 \%$ nonlinearity of (5). At this time, the algebraic system is almost considered as a linear system. The value of nonlinearity index which equals one indicates a percent worst-case departure from the linear approximation.

2.2. Nonlinear Measurement for Dynamical Systems. Consider a dynamical model

$$
\begin{aligned}
\dot{x} & =f(x, t), \\
x\left(t_{0}\right) & =x_{0},
\end{aligned}
$$

where $f(x, t)$ is a differentiable function.

A reference initial state $\widetilde{x}\left(t_{0}\right)=\widetilde{x}_{0}$ is defined. Then the appropriate state transition matrix for $\widetilde{x}\left(t_{0}\right)$ with time $t$ is computed as

$$
\widetilde{\Phi}(t)=\frac{\widetilde{x}(t)}{\widetilde{x}\left(t_{0}\right)}=\frac{d \widetilde{x}}{d \widetilde{x}_{0}},
$$

where $\widetilde{x}(t)=\widetilde{x}$ is a state at any given time $t$ and $\widetilde{\Phi}(t)$ is the state transition matrix of the reference initial state.

The state transition matrix $\widetilde{\Phi}(t)$ should satisfy the following equations:

$$
\begin{aligned}
\dot{\Phi}(t) & =\widetilde{A}(t) \widetilde{\Phi}(t), \\
\widetilde{A}(t) & =\frac{\partial f(x, t)}{\partial x}, \\
\widetilde{\Phi}\left(t_{0}\right) & =I .
\end{aligned}
$$

In the neighbourhood of the reference state $\widetilde{x}\left(t_{0}\right)=\widetilde{x}_{0}$, there is a family of initial condition variations: $x_{0}=\widetilde{x}_{0}+\delta x_{0}$.

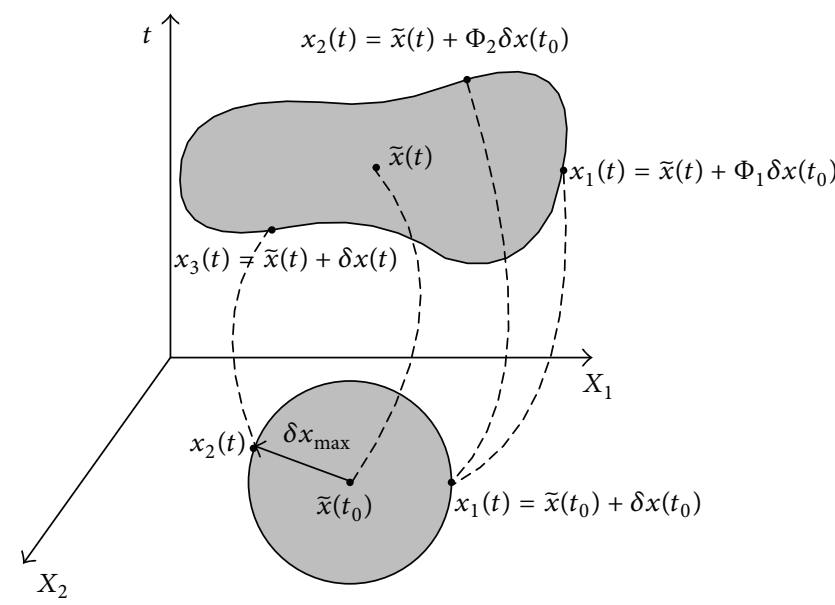

FIGURE 4: The idea of nonlinearity index of a dynamic model.

The region $\left\|\delta x_{0}\right\| \leq \delta x_{\max }$ limits the range of these variations. These variations satisfy the following differential equations:

$$
\begin{aligned}
\dot{x} & =f(x, t), \\
x\left(t_{0}\right) & =\tilde{x}_{0}+\delta x_{0}, \\
\left\|\delta x_{0}\right\| & \leq \delta x_{\max } .
\end{aligned}
$$

The corresponding state transition matrix is

$$
\Phi(t)=\frac{x(t)}{x\left(t_{0}\right)}=\frac{d x}{d x_{0}} .
$$

Equation (10) satisfies the following expressions:

$$
\begin{aligned}
\dot{\Phi}(t) & =A(t) \Phi(t), \\
A(t) & =\frac{\partial f(x, t)}{\partial x}, \\
\Phi\left(t_{0}\right) & =I .
\end{aligned}
$$

For measuring the nonlinear strength of a dynamic model, the nonlinearity index is given by

$$
v(t)=\sup \frac{\|\Phi(t)-\widetilde{\Phi}(t)\|}{\|\widetilde{\Phi}(t)\|} .
$$

In the dynamical model, the nonlinear initial problem is concerned. The nonlinearity index measures the relative variation of the state transition matrix. The maximum deviation between $\Phi(t)$ and the reference $\widetilde{\Phi}(t)$ embodies the nonlinear degree of dynamics model. Figure 4 shows the idea of nonlinearity index of dynamical model. The nonlinearity index has a different value at point $x_{2}$ over the time interval $\left(t-t_{0}\right)$. If the model is linear, the trajectory of an initial point is the same one, and the nonlinearity index is zero.

The nonlinearity index for dynamical model is allied to the Lyapunov exponent. The nonlinearity index is considered as the average rate of convergence or divergence of different 
trajectories from the same point. The corresponding expression is as follows:

$$
\lambda_{i}=\lim _{t \rightarrow \infty} \frac{1}{t} \frac{\left|\delta x_{i}(t)\right|}{\left|\delta x_{i}\left(t_{0}\right)\right|}=\lim _{t \rightarrow \infty} \frac{1}{t}\left|\Phi_{i}(t)\right|, \quad i=1,2, \ldots
$$

If $\Lambda$ is defined as the maximum of all possible Lyapunov exponent, the expression of $\Lambda$ can be written by

$$
\Lambda=\lim _{t \rightarrow \infty} \frac{1}{t} \ln \|\Phi(t)\|_{2} .
$$

Then, the nonlinearity index for dynamics model can also be expressed as follows:

$$
\lim _{t \rightarrow \infty} v(t) \sim \sup \frac{e^{\Lambda}-e^{\widetilde{\Lambda}}}{e^{\widetilde{\Lambda}}} .
$$

The main disadvantage of Lyapunov exponent is that the infinite time limit is defined. Therefore, in the short time, the rate of convergence or divergence is not realized. So the nonlinearity index is an effective method to measure the rate of convergence or divergence for different parts of trajectory.

2.3. Nonlinear Measurement for State-Space Systems. Consider a general nonlinear dynamic model

$$
\begin{aligned}
& \dot{x}=f(x, u, t), \\
& y=g(x, u, t),
\end{aligned}
$$

where $x$ is the state vector, $y$ is the output vector, $u$ is the input vector, and $f(\cdot)$ and $g(\cdot)$ are the nonlinear functions.

A feature point $\hat{x}$ is chosen. At the point $\hat{x}$, a linearized model is got in lieu of (16). The linear model can be expressed as a state-space model. The state-space model is described as

$$
\begin{aligned}
\delta \dot{x} & =\widehat{A} \delta x+\Delta_{u} f \delta u, \\
\delta y & =\widehat{C} \delta x+\widehat{D} \delta u, \\
\widehat{A} & =\left.\Delta_{x} f\right|_{x=\widehat{x}}, \\
\widehat{B} & =\left.\Delta_{u} f\right|_{x=\widehat{x}}, \\
\widehat{C} & =\left.\Delta_{x} g\right|_{x=\widehat{x}}, \\
\widehat{D} & =\left.\Delta_{u} g\right|_{x=\widehat{x}},
\end{aligned}
$$

where $\Delta_{x} f$ and $\Delta_{u} f$ denote first partial derivatives of the function $f(\cdot)$ with respect to $x$ and $u$. $\Delta_{x} g$ and $\Delta_{u} g$ denote first derivatives of the function $g(\cdot)$ with respect to $x$ and $u$.

A certain vicinity around $\widehat{x}$ is defined. In this region, the variation $x=\widehat{x}+\delta x,\|\delta x\| \leq \delta x_{\max }$. The $\delta x$ represents a bound variation about the feature point. The corresponding linear model can be written as

$$
\begin{aligned}
\delta \dot{x} & =A \delta x+B \delta u, \\
\delta y & =C \delta x+D \delta u, \\
A & =\left.\Delta_{x} f\right|_{x=\widehat{x}+\delta x}, \\
B & =\left.\Delta_{u} f\right|_{x=\widehat{x}+\delta x}, \\
C & =\left.\Delta_{x} g\right|_{x=\widehat{x}+\delta x}, \\
D & =\left.\Delta_{u} g\right|_{x=\hat{x}+\delta x} .
\end{aligned}
$$

As (5), the four static nonlinearity indices are expressed as follows:

$$
\begin{aligned}
& v_{s}^{A}=\sup \frac{\|A-\bar{A}\|}{\|\bar{A}(t)\|}, \\
& v_{s}^{B}=\sup \frac{\|B-\bar{B}\|}{\|\bar{B}\|}, \\
& v_{s}^{C}=\sup \frac{\|C-\bar{C}\|}{\|\bar{C}\|}, \\
& v_{s}^{D}=\sup \frac{\|D-\bar{D}\|}{\|\bar{D}\|},
\end{aligned}
$$

where $v_{s}^{A}, v_{s}^{B}, v_{s}^{C}$, and $v_{s}^{D}$ are the static state, static input, static output, and static direct nonlinearity indices. The indices $v_{s}^{A}$ and $v_{s}^{C}$ address the nonlinear feature of system related to initial condition excitation, whereas the static indices $v_{s}^{B}$ and $v_{s}^{D}$ address the nonlinear feature of system related to input excitation.

Taking into account the dynamic process of the model, the four nonlinearity indices are given by

$$
\begin{aligned}
v_{d}^{x}(t) & =\sup \frac{\|\Phi(t)-\widehat{\Phi}(t)\|}{\|\widehat{\Phi}(t)\|}, \\
v_{d}^{u}(t) & =\sup \frac{\|\Phi(t) B-\widehat{\Phi}(t) \widehat{B}\|}{\|\widehat{\Phi}(t) \widehat{B}\|}, \\
v_{d}^{y}(t) & =\sup \frac{\|C(t) \Phi(t)-\widehat{C} \widehat{\Phi}(t)\|}{\|\widehat{C} \widehat{\Phi}(t)\|}, \\
v_{d}^{y / u}(t) & =\sup \frac{\|C \Phi(t) B-\widehat{C} \widehat{\Phi}(t) \widehat{B}\|}{\|\widehat{C} \widehat{\Phi}(t) \hat{B}\|},
\end{aligned}
$$

where $v_{d}^{x}$ is the dynamic state, $v_{d}^{u}$ is the dynamic input, $v_{d}^{y}$ is the dynamic output, $v_{d}^{y / u}$ is the dynamic input-output nonlinearity indices, $\Phi(t)$ is the transition matrix for $A$, and $\widehat{\Phi}(t)$ is the transition matrix for $\bar{A}$. 
The static and dynamic nonlinearity indices not only can qualitatively and quantitatively analyze the nonlinear strength of any models but also can indicate the source of nonlinearity.

2.4. Extension to Flight Dynamical Systems. In this part, the nonlinearity index theory will be extended to the flight dynamical systems. The equations of nonlinear dynamics can be described as

$$
\dot{x}=A(\sigma(t)) x+B(\sigma(t)) u,
$$

where $\sigma(t)$ is the time-varying parameter vector.

If $\sigma(t)$ is also the state of system, the system is called a quasi-linear-parameter-varying system.

The Jacobian linearization approach is applied to (21). The quasi-linear-parameter-varying model of nonlinear dynamics is written by

$$
\delta \dot{x}=A(\sigma(t)) \delta x+B(\sigma(t)) \delta u .
$$

At a family of feature points $x_{j}, j=1,2, \ldots$, the quasi-LPV model is defined by

$$
\delta \dot{x}=A_{j}\left(\sigma_{j}(t)\right) \delta x+B_{j}\left(\sigma_{j}(t)\right) \delta u .
$$

A certain vicinity around $x_{j}$ is defined. In this range, there are a set of points $x_{i}=x_{j}+\delta x_{j}, i=1,2, \ldots$. The $\delta x_{j}$ is the bound variation, $\left\|\delta x_{j}\right\| \leq \delta x_{\max }$. The corresponding quasiLPV model can be described as follows:

$$
\delta \dot{x}=A_{i}\left(\sigma_{i}(t)\right) \delta x+B_{i}\left(\sigma_{i}(t)\right) \delta u .
$$

The nonlinearity indices of flight dynamical systems can be expressed as follows:

$$
\begin{aligned}
& v_{s}^{A}\left(x_{j}\right)=\sup _{\substack{i=1,2,3, \ldots \\
j=1,2,3, \ldots}} \frac{\left\|A_{i}\left(\sigma_{i}\right)-A_{j}\left(\sigma_{j}\right)\right\|}{\left\|A_{j}\left(\sigma_{j}\right)\right\|}, \\
& v_{s}^{B}\left(x_{j}\right)=\sup _{\substack{i=1,2,3, \ldots \\
j=1,2,3, \ldots}} \frac{\left\|B_{i}\left(\sigma_{i}\right)-B_{j}\left(\sigma_{j}\right)\right\|}{\left\|\bar{B}_{j}\left(v_{j}\right)\right\|}, \\
& v_{d}^{x}\left(t, x_{j}\right)=\sup _{\substack{i=1,2,3, \ldots \\
j=1,2,3, \ldots}} \frac{\left\|\Phi_{i}\left(v_{i}\right)-\Phi_{j}\left(\sigma_{j}\right)\right\|}{\left\|\bar{\Phi}_{j}\left(\sigma_{j}\right)\right\|}, \\
& v_{d}^{u}\left(t, x_{j}\right) \underset{\substack{i=1,2,3, \ldots . \\
j=1,2,3, \ldots}}{\left\|\Phi_{i}\left(\sigma_{i}\right) B_{i}\left(\sigma_{i}\right)-\Phi_{j}\left(\sigma_{j}\right) B_{j}\left(\sigma_{j}\right)\right\|} \\
& \left\|\Phi_{j}\left(\sigma_{j}\right) B_{j}\left(\sigma_{j}\right)\right\|
\end{aligned}
$$

where $v_{s}^{A}, v_{s}^{B}, v_{d}^{x}$, and $v_{d}^{u}$ are the static state, static input, dynamic state, and dynamic input nonlinearity indices on the feature points $x_{j}$, respectively.

The four nonlinearity indices indicate the maximum deviation between the feature point and a set of points around it in different ways. The static state nonlinearity index $v_{s}^{A}$ is for the state matrix $A$. The dynamic state nonlinearity index $v_{d}^{x}$ measures the propagation of state matrix $A$ with time. The $v_{s}^{B}$ and $v_{d}^{u}$ express the static and dynamic influences on complex systems with the input signal.

For analysing the dynamical model, the parameter vector $\sigma(t)$ should be chosen to capture the nonlinear feature of complex systems. In this paper, to meet the real-world engineering conditions, the scheduling parameters are angle of attack and Mach number $\sigma=\left[\begin{array}{ll}\alpha & \mathrm{Ma}\end{array}\right]$.

Being state vector, angle of attack and Mach number should be used as parameters to calculate the nonlinearity index, respectively. Two expressions of nonlinearity index can be given as

$$
\begin{aligned}
& v_{s}^{A}\left(\alpha_{j}\right)=\sup _{\substack{i=1,2,3, \ldots \\
j=1,2,3, \ldots}} \frac{\left\|A_{i}\left(\alpha_{i}, \mathrm{Ma}\right)-A_{j}\left(\alpha_{j}, \mathrm{Ma}\right)\right\|}{\left\|A_{j}\left(\sigma_{j}\right)\right\|}, \\
& v_{s}^{B}\left(\alpha_{j}\right)=\sup _{\substack{i=1,2,3, \ldots \\
j=1,2,3, \ldots}} \frac{\left\|B_{i}\left(\alpha_{i}, \mathrm{Ma}\right)-B_{j}\left(\alpha_{j}, \mathrm{Ma}\right)\right\|}{\left\|\bar{B}_{j}\left(\sigma_{j}\right)\right\|}, \\
& v_{d}^{x}\left(t, \alpha_{j}\right)=\sup _{\substack{i=1,2,3, \ldots \\
j=1,2,3, \ldots}} \frac{\left\|\Phi_{i}\left(\alpha_{i}, \mathrm{Ma}\right)-\Phi_{j}\left(\alpha_{j}, \mathrm{Ma}\right)\right\|}{\left\|\bar{\Phi}_{j}\left(\alpha_{j}, \mathrm{Ma}\right)\right\|}, \\
& v_{d}^{u}\left(t, \alpha_{j}\right) \\
& =\sup _{\substack{i=1,2,3, \ldots \\
j=1,2,2, \ldots}} \frac{\left\|\Phi_{i}\left(\alpha_{i}, \mathrm{Ma}\right) B_{i}\left(\alpha_{i}, \mathrm{Ma}\right)-\Phi_{j}\left(\alpha_{j}, \mathrm{Ma}\right) B_{j}\left(\alpha_{j}, \mathrm{Ma}\right)\right\|}{\left\|\Phi_{j}\left(\alpha_{j}, \mathrm{Ma}\right) B_{j}\left(\alpha_{j}, \mathrm{Ma}\right)\right\|}, \\
& v_{s}^{A}\left(\mathrm{Ma}_{j}\right)=\sup _{\substack{i=1,2,3, \ldots \\
j=1,2,3, \ldots}} \frac{\left\|A_{i}\left(\alpha, \mathrm{Ma}_{i}\right)-A_{j}\left(\alpha, \mathrm{Ma}_{j}\right)\right\|}{\left\|A_{j}\left(\alpha, \mathrm{Ma}_{j}\right)\right\|}, \\
& v_{s}^{B}\left(\mathrm{Ma}_{j}\right)=\sup _{\substack{i=1,2,3, \ldots \\
j=1,2,3, \ldots}} \frac{\left\|B_{i}\left(\alpha, \mathrm{Ma}_{i}\right)-B_{j}\left(\alpha, \mathrm{Ma}_{j}\right)\right\|}{\left\|\bar{B}_{j}\left(\alpha, \mathrm{Ma}_{j}\right)\right\|}, \\
& v_{d}^{x}\left(t, \mathrm{Ma}_{j}\right)=\sup _{\substack{i=1,2,3, \ldots \\
j=1,2,3, \ldots}} \frac{\left\|\Phi_{i}\left(\alpha, \mathrm{Ma}_{i}\right)-\Phi_{j}\left(\alpha, \mathrm{Ma}_{j}\right)\right\|}{\left\|\bar{\Phi}_{j}\left(\alpha, \mathrm{Ma}_{j}\right)\right\|}, \\
& v_{d}^{u}\left(t, \mathrm{Ma}_{j}\right) \\
& =\sup _{\substack{i=1,2,3, \ldots \\
j=1,2,3, \ldots}} \frac{\left\|\Phi_{i}\left(\alpha, \mathrm{Ma}_{i}\right) B_{i}\left(\alpha, \mathrm{Ma}_{i}\right)-\Phi_{j}\left(\alpha, \mathrm{Ma}_{j}\right) B_{j}\left(\alpha, \mathrm{Ma}_{j}\right)\right\|}{\left\|\Phi_{j}\left(\alpha, \mathrm{Ma}_{j}\right) B_{j}\left(\alpha, \mathrm{Ma}_{j}\right)\right\|} .
\end{aligned}
$$

Therefore, at the feature point $\sigma_{j}$, the static and dynamic nonlinearity indices are expressed as

$$
\begin{aligned}
& v_{s}^{A}\left(\sigma_{j}\right)=\sqrt{\left(v_{s}^{A}\left(\alpha_{j}\right)\right)^{2}+\left(v_{s}^{A}\left(\mathrm{Ma}_{j}\right)\right)^{2}}, \\
& v_{s}^{B}\left(\sigma_{j}\right)=\sqrt{\left(v_{s}^{B}\left(\alpha_{j}\right)\right)^{2}+\left(v_{s}^{B}\left(\mathrm{Ma}_{j}\right)\right)^{2}}, \\
& v_{d}^{x}\left(\sigma_{j}\right)=\sqrt{\left(v_{d}^{x}\left(\alpha_{j}\right)\right)^{2}+\left(v_{d}^{x}\left(\mathrm{Ma}_{j}\right)\right)^{2}}, \\
& v_{d}^{u}\left(\sigma_{j}\right)=\sqrt{\left(v_{d}^{u}\left(\alpha_{j}\right)\right)^{2}+\left(v_{d}^{u}\left(\mathrm{Ma}_{j}\right)\right)^{2}} .
\end{aligned}
$$




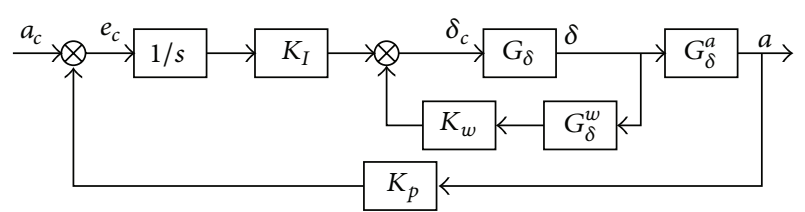

FIGURE 5: Controller structure.

\section{Controller Design Based on Nonlinear Strength}

3.1. Controller Design. From [6], we know that open-loop control systems are sensitive to external disturbances or modeling uncertainties. For the missile controller design, the closed-loop control systems are always used. A closedloop control law should be of the lowest possible complexity for implementation reasons and, in addition, at some level, should be able to adapt to the changing operating conditions of the system such as altitude and airspeed.

A simple structure of a control law such as proportional integral and cascaded proportional/proportional integral has been used on missile autopilot systems in [7-11] and is a particularly well-suited practice for these systems. The reason is that the controller has a low complexity, a high robustness, and a strong applicability on the full-order or nonlinear control system. This paper employs a gain scheduling control method and a closed-loop control law to design the missile control system.

An overview of the proposed missile control system is shown in Figure 5. The control structure has three loops: In the inner loop, the control gain $K_{w}$ is applied to the angular rates and adds damping; another one, $K_{p}$, is applied to the signals and tailors the system time performance; finally, one $K_{I}$ is applied to the tracking error signals and provides steadystate tracking accuracy.

The basic theory of the gain scheduling control method is very accessible to us. In the aspect of missile control approaches, gain scheduling is always one of the popular engineering methods. The overview of the gain scheduling method combining with nonlinearity index theory is shown in Figure 6.

3.2. Nonlinearity Strength Scale. The gain-scheduled control method needs to grid down the flight envelope to a set of feature points in each subregion, for which the system performance is supposed to be identical. Many researches [1216] provide several sophisticated methods such as Lyapunov stability theory for analyzing the control performance in the subregion. These methods show a capability to expose and understand the stability of the control system. However, neither of such methods can qualitative and quantify the nonlinearity of the control system in the subregion, which verify the control parameter of the feature point to be fit for all the subregion condition. The controller design based on nonlinearity index theory will provide a new idea to solve this question. The main idea of the method is shown in Figure 7.

The static nonlinearity indices $v_{s}^{A}, v_{s}^{B}$ measure the nonlinear strength of state variables and input signals of

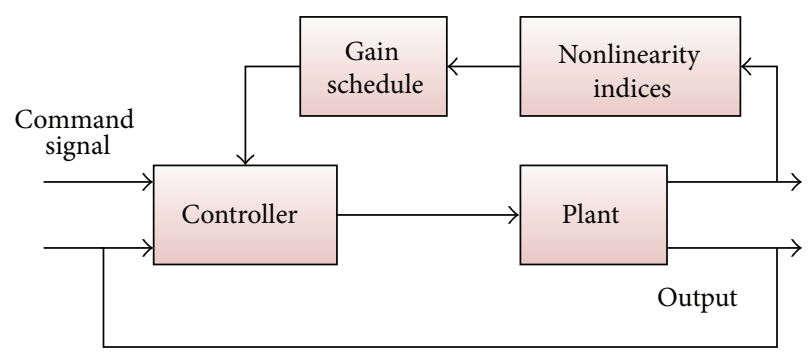

FIGURE 6: Gain scheduling sketch.

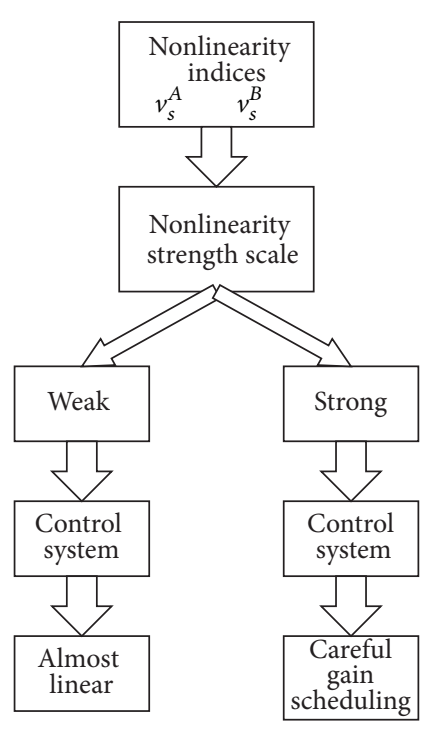

FIGURE 7: Controller design based on nonlinearity indices.

the missile linear model, respectively. According to the static nonlinearity indices, the nonlinearity strength scale is scoped. As the gain scheduling control parameter, we must quantitatively and qualitatively analyze the nonlinearity strength scale. When the nonlinearity indices approximate or equal zero, the missile behavior is closed to a linear system, and a feature point can represent the control characteristic of this nonlinearity region. However, a higher value of the static nonlinearity indices accounts for the strong nonlinear nature over the worst-case region, so more feature points must be chosen to analyze the control characteristic in this region.

3.3. Sample Time Selection. The dynamic nonlinearity index $v_{d}^{x}$ measures the propagation of state nonlinearity with the fundamental sample time of simulation, and the index $v_{d}^{u}$ represents the influence of input signal with the sample time. From (25), we know that the sample time has a great impact on the dynamic nonlinearity indices, a higher value making a stronger nonlinearity of the missile system. A small fixedstep size can improve the precision of linearization methods, but huge computation is the limitation. So an adaptive sample time, which can be got by analyzing the dynamic nonlinearity indices, is very important to generate a fit nonlinearity strength scale and convenient calculation. 


\section{Simulation}

4.1. Nonlinear Analysis. In this paper, the missile is axisymmetric, so the absolute value of the longitudinal and lateral aerodynamic coefficient is the same. Through analyzing, the nonlinearity of the missile is from the aerodynamic force and external force moment. Even though the force and moment decomposition appears to be linear, the aerodynamic coefficients present a heavily nonlinear form with respect to the attack angle and Mach number.

Explicit limits correspond to control input saturation or aerodynamic angles on wind tunnel experiment and for the missile model correspond to

$$
\begin{gathered}
-12 \mathrm{deg} \leq \alpha \leq 12 \mathrm{deg} \\
-15 \mathrm{deg} \leq \delta \leq 15 \mathrm{deg} \\
0.8 \leq \mathrm{Ma} \leq 1.2
\end{gathered}
$$

Now we introduce the nonlinearity generated by aerodynamic coefficients. From [17, 18], aerodynamics of missile is analyzed. In the simulation analysis, considering the information of wind tunnel experiment and axisymmetric missile, we just calculate the situation that aerodynamic angles are greater than zero. An example illustrating this nonlinearity is given in Figure 8, showing the resistance coefficient surface $\mathrm{C}_{x}(\mathrm{Ma}, \alpha)$.

4.2. Nonlinearity Index for Missile Dynamics Assessment. In the present development, the missile has six degrees of freedom (6-DOF) consisting of three translations and three rotations. In [19], the translation and rotation of the missile may be expressed mathematically by the following equations:

$$
\begin{aligned}
m \frac{\mathrm{d} \mathbf{V}}{\mathrm{d} t} & =\mathbf{P}+\mathbf{G}+\mathbf{R}, \\
\frac{\mathrm{d} \mathbf{H}}{\mathrm{d} t} & =\mathbf{M},
\end{aligned}
$$

where $\mathbf{P}$ denotes the engine thrust vector, $\mathbf{G}$ denotes the gravity vector of the missile, $\mathbf{R}$ denotes aerodynamic force vector, $\mathbf{M}$ denotes the external force moment vector, and $\mathbf{H}$ denotes the angular momentum vector.

The full-envelope motion of a missile can be fairly well described by a longitudinal motion and a lateral-directional motion. Because of the axisymmetric missile in the paper, only considering a longitudinal motion is enough to describe the missile motion. In the case of the symmetric longitudinal flight, the lateral-directional variables are exactly zero. The operating attack angle $\alpha$ and Mach number Ma have been selected herein to construct the operating condition parameter vector $\lambda=[\mathrm{Ma} \alpha]$. Given this operating condition parameter vector $\lambda$, the states' and inputs' trimmed values are obtained by solving a set of nonlinear algebraic equations [20]. Changing the operating condition parameter vector

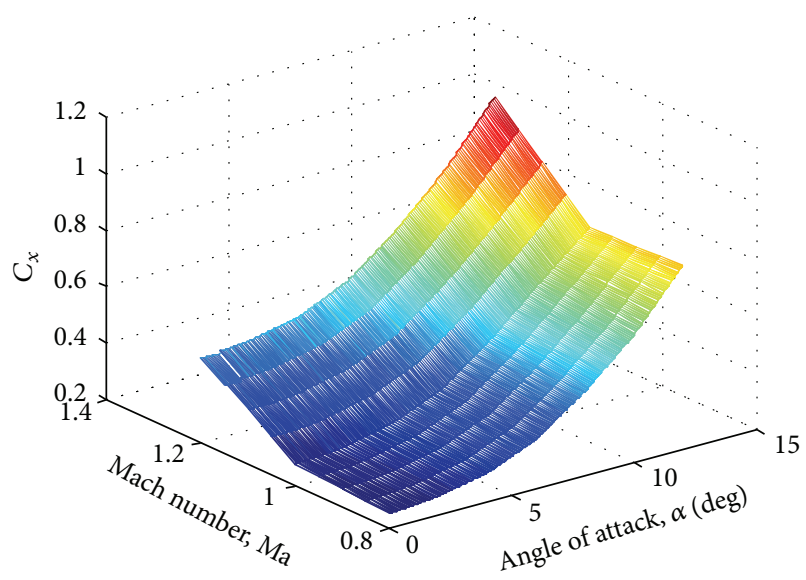

FIGURE 8: Resistance coefficient surface.

causes changes to the equivalent trimmed values and linear model. The linear model of the longitudinal motion is

$$
\begin{aligned}
& x=A x+B u, \\
& u=\delta_{z}, \\
& x=\left[\begin{array}{c}
\Delta V \\
\Delta \omega_{z} \\
\Delta \alpha \\
\Delta \vartheta
\end{array}\right],
\end{aligned}
$$

where

A

$$
\begin{aligned}
& =\left[\begin{array}{cccc}
-\frac{q S}{m} \frac{\partial c_{x}}{\partial V} & 0 & -\frac{q S}{m} \frac{\partial c_{x}}{\partial \alpha}+g \cos \theta & -g \cos \theta \\
\frac{q S l}{J_{z}} \frac{\partial m_{z}(\alpha)}{\partial V} & \frac{q S l m_{z}^{\omega_{z}}}{J_{z}} & \frac{1}{J_{z}} \frac{\partial m_{z}(\alpha)}{\partial \alpha} & 0 \\
\frac{q S}{m V} \frac{\partial c_{y}(\alpha)}{\partial V} & 1 & -\frac{q S}{m V} \frac{\partial c_{y}(\alpha)}{\partial a}+\frac{g \sin \theta}{V} & -\frac{g \sin \theta}{V} \\
0 & 1 & 0 & 0
\end{array}\right] \\
& \bar{B}=\left[\begin{array}{c}
-\frac{q S}{m} \frac{\partial c_{x}}{\partial \delta_{z}} \\
\frac{q S l}{I_{z}} \frac{\partial m_{z}\left(\delta_{z}\right)}{\partial \delta_{z}} \\
-\frac{q S}{m V} \frac{\partial c_{y}\left(\delta_{z}\right)}{\partial \delta_{z}} \\
0
\end{array}\right] .
\end{aligned}
$$

$q$ is dynamic pressure. $S$ is characteristic area. $l$ is characteristic length. $c_{x}$ and $c_{y}$ are drag and lift force coefficients. $m_{z}$ is pitching moment coefficient.

In the engineering, the data of aerodynamic coefficients is given by the look-up tables (LUT). The aerodynamic coefficients are considered as an interpolation function of angle of attack and Mach number. Therefore, in (30), the derivative of the aerodynamic coefficients with respect to angle of attack, flight velocity, and rudder deflection, respectively, should be 
calculated by the deviation of two interpolating points. The corresponding expression can be described as

$$
\begin{aligned}
\frac{\partial c_{x}}{\partial V} & =\frac{c_{x}\left(\sigma_{i+1}\right)-c_{x}\left(\sigma_{i}\right)}{V_{i+1}-V_{i}} \\
\frac{\partial c_{x}}{\partial \alpha} & =\frac{c_{x}\left(\sigma_{i+1}\right)-c_{x}\left(\sigma_{i}\right)}{\alpha_{i+1}-\alpha_{i}} \\
\frac{\partial m_{z}(\alpha)}{\partial V} & =\frac{m_{z}^{\alpha}\left(\sigma_{i+1}\right)-m_{z}^{\alpha}\left(\sigma_{i}\right)}{V_{i+1}-V_{i}} \\
\frac{\partial m_{z}(\alpha)}{\partial \alpha} & =\frac{m_{z}^{\alpha}\left(\sigma_{i+1}\right)-m_{z}^{\alpha}\left(\sigma_{i}\right)}{\alpha_{i+1}-\alpha_{i}} \\
\frac{\partial c_{y}(\alpha)}{\partial V} & =\frac{c_{y}^{\alpha}\left(\sigma_{i+1}\right)-c_{y}^{\alpha}\left(\sigma_{i}\right)}{V_{i+1}-V_{i}} \\
\frac{\partial c_{y}(\alpha)}{\partial \alpha} & =\frac{c_{y}^{\alpha}\left(\sigma_{i+1}\right)-c_{y}^{\alpha}\left(\sigma_{i}\right)}{\alpha_{i+1}-\alpha_{i}} \\
\frac{\partial m_{z}\left(\delta_{z}\right)}{\partial \delta_{z}} & =\frac{m_{z}^{\delta_{z}}\left(\sigma_{i+1}\right)-m_{z}^{\delta_{z}}\left(\sigma_{i}\right)}{\Delta \delta_{z}} \\
\frac{\partial c_{y}\left(\delta_{z}\right)}{\partial \delta_{z}} & =\frac{c_{y}^{\delta_{z}}\left(\sigma_{i+1}\right)-c_{y}^{\delta_{z}}\left(\sigma_{i}\right)}{\Delta \delta_{z}} \\
& =\frac{c_{x}\left(\sigma_{i+1}\right)-c_{x}\left(\sigma_{i}\right)}{\Delta \delta_{z}} .
\end{aligned}
$$

According to (30), a nominal linear model with a notation $\left\{\bar{A}_{j}, \bar{B}_{j}\right\}$ is developed. In the worst-case region around the point $j$, a set of points, $i=1,2, \ldots, N$, is seeded and an equivalent set of linear systems $\left\{\bar{A}_{i}, \bar{B}_{i}\right\}$ is generated. Now directly applying the four expressions in (27) to the missile models leads to a set of surfaces. Visualization of such surfaces enables nonlinearity to be expressed more intuitively. The static nonlinearity indices $v_{s}^{A}$ and $v_{s}^{B}$ of the missile model are shown in Figures 9 and 10.

The dynamics indices $v_{d}^{x}$ and $v_{d}^{u}$ are not only about the scheduling parameters but also about time. The dynamic nonlinearity indices at time $t=0.01 \mathrm{~s}$ are shown in Figures 11 and 12 . The dynamic nonlinearity indices at time $t=0.1 \mathrm{~s}$ are shown in Figures 13 and 14. Comparing with Figures 11 and 13, the simulation step size dominates the deviation between the linearized model and nonlinear model. The large deviations mean a high value of dynamic nonlinearity index $v_{d}^{x}$ and lead to a strong nonlinear strength of dynamical model. Comparing with Figures 12 and 14, the change of dynamic input nonlinearity index $v_{d}^{u}$ is small. The nonlinear strength of dynamical model due to the input vectors is lower than the state vectors.

From Figures 9-14, the missile motion shows a strong nonlinearity characteristic. The nonlinearity strength scale is divided into four parts: Part $1, S_{1}=\{(\alpha, \mathrm{Ma}) \mid \alpha \epsilon$ $\left.\left[0,3^{\circ}\right], \mathrm{Ma} \in[0.8,1.2]\right\} ;$ Part 2, $S_{2}=\{(\alpha, \mathrm{Ma}) \mid \alpha \in$ $\left.\left[3^{\circ}, 10^{\circ}\right], \mathrm{Ma} \in[0.8,1]\right\} ;$ Part $3, S_{3}=\{(\alpha, \mathrm{Ma}) \mid \alpha \epsilon$ $\left.\left[3^{\circ}, 12^{\circ}\right], \mathrm{Ma} \in[1,1.2]\right\} ; \operatorname{Part} 4, S_{4}=\{(\alpha, \mathrm{Ma}) \mid \alpha \epsilon$ $\left[10^{\circ}, 12^{\circ}\right]$, Ma $\left.\in[0.8,1]\right\}$. According to the nonlinearity

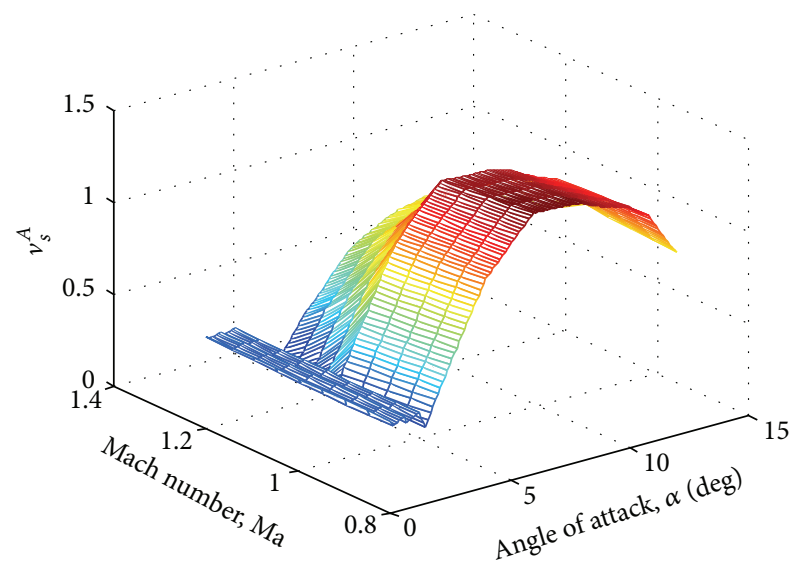

FIGURE 9: Static state nonlinearity index.

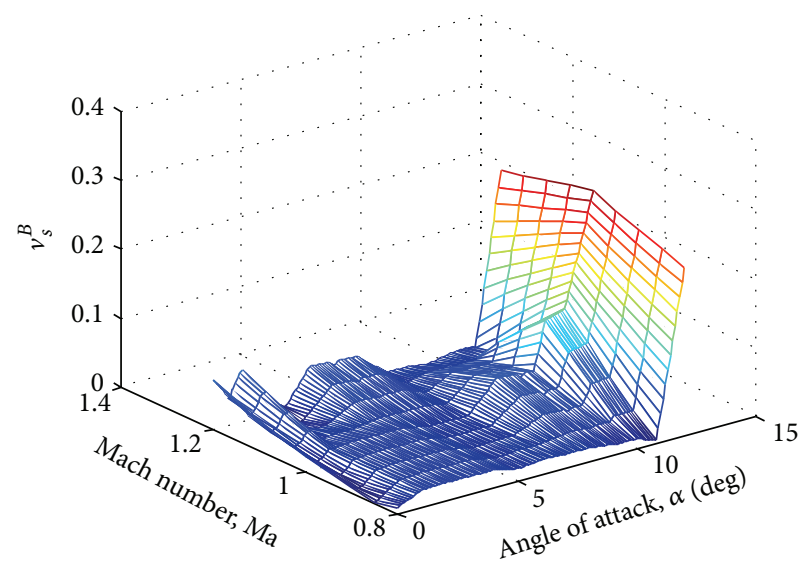

FIGURE 10: Static input nonlinearity index.

strength and the computational performance, the fundamental sample time of simulation is equal to $0.05 \mathrm{~s}$.

4.3. Analysis of Control Characteristic. According to the difference between strong and weak nonlinearities, the varying parameter control system is designed. Figures 12-15 show the time domain characteristic of the four parts, which are addressed in the above section. Moreover, to test the method of this paper still further, 6-DOF full nonlinear simulation results of the overall guidance and control configuration are given. In accordance with these simulation results, the control method proposed in this paper can fairly well realize the attitude tracking control and keep the attitude stabilization.

In the region of Part 1 , at the point $(\alpha=2, \mathrm{Ma}=1) \in S_{1}$, the control parameter is $K_{p}=28.8, K_{i}=1.9$, and $K_{w}=6.5$. The step response is shown in Figure 15, of which the rise time is $0.6 \mathrm{~s}$ and the overshoot is $13.4 \%$. The Bode diagram is shown in Figure 16, in which GM is infinity and PM is $55.2 \mathrm{deg}$. The PID controller is designed to improve system dynamic performance.

In the region of Part 2, at the point $(\alpha=6, \mathrm{Ma}=$ 0.9) $\in S_{2}$, the control parameter is $K_{p}=20.8, K_{i}=1.9$, and $K_{w}=5.0$. The step response is shown in Figure 17, of 


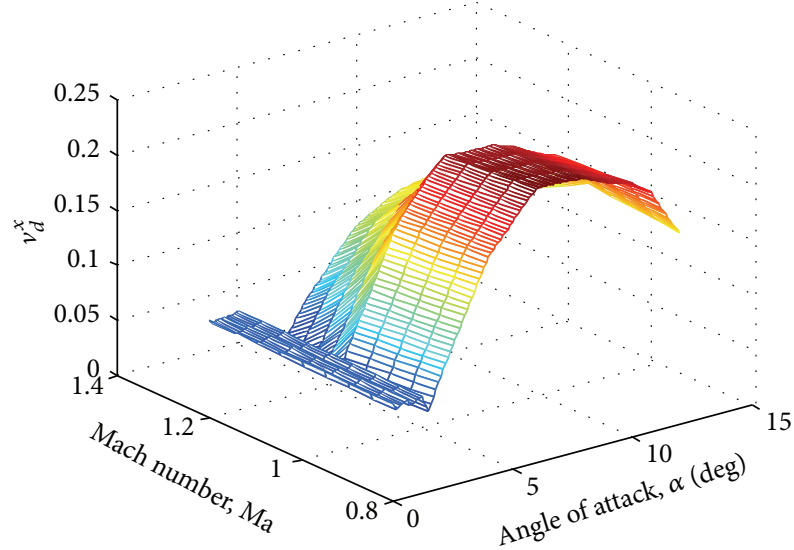

FIGURE 11: Dynamic state nonlinearity index.

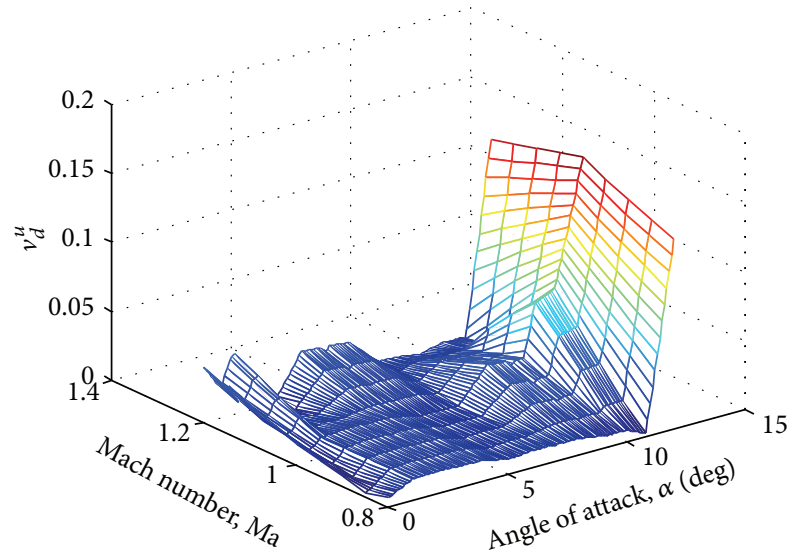

Figure 12: Dynamic input nonlinearity index.

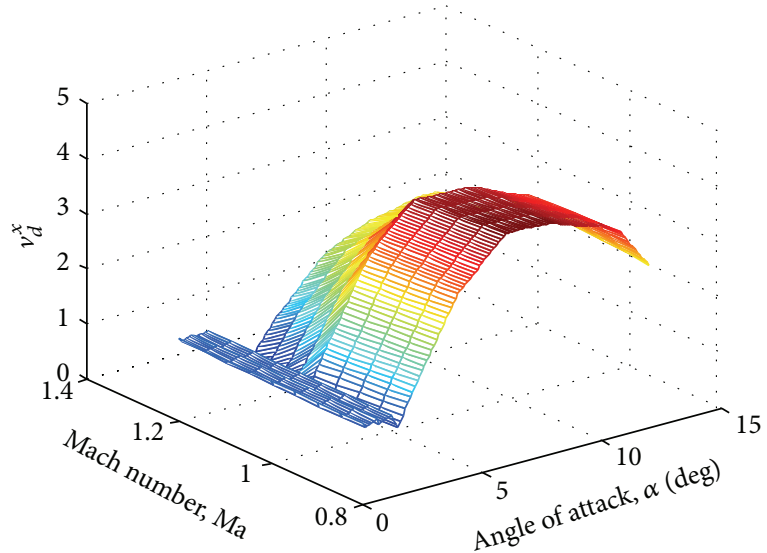

FIGURE 13: Dynamic state nonlinearity index.

which the rise time is $0.66 \mathrm{~s}$ and the overshoot is $14.4 \%$. The Bode diagram is shown in Figure 18, in which GM is infinity and PM is $54.7 \mathrm{deg}$. The control system response meets the requirements of control system.

In the region of Part 3, at the point $(\alpha=8, \mathrm{Ma}=1.2) \epsilon$ $S_{3}$, the control parameter is $K_{p}=12.8, K_{i}=1.9$, and $K_{w}=$

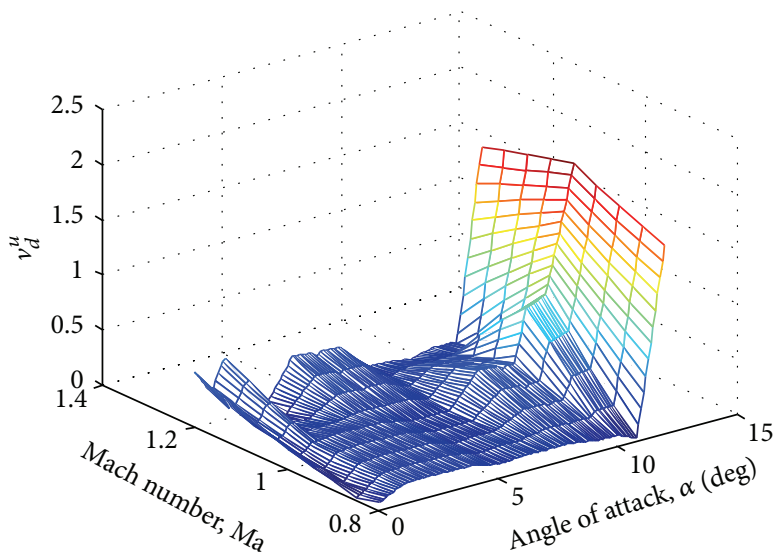

FIGURE 14: Dynamic input nonlinearity index.

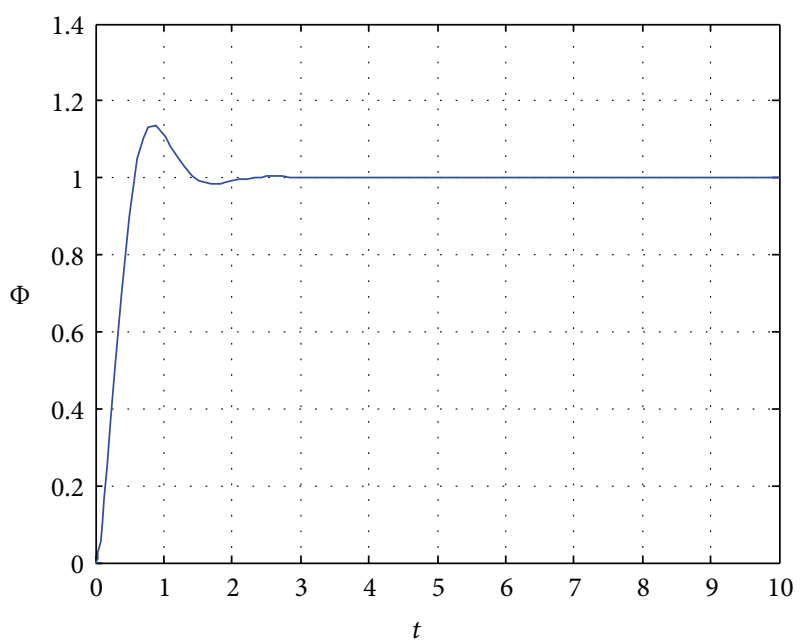

Figure 15: Step response on Part 1.
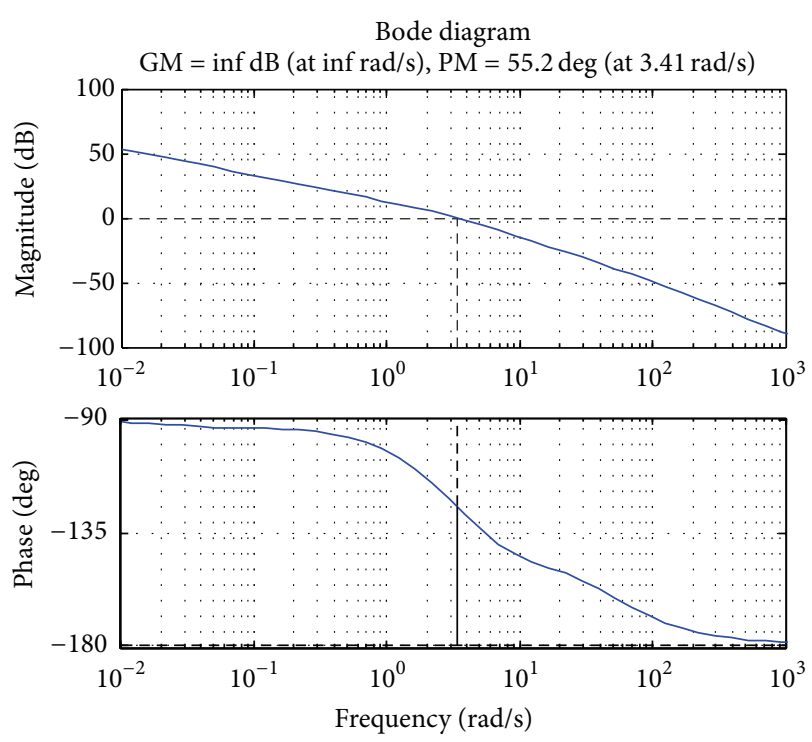

Figure 16: Bode diagram on Part 1. 


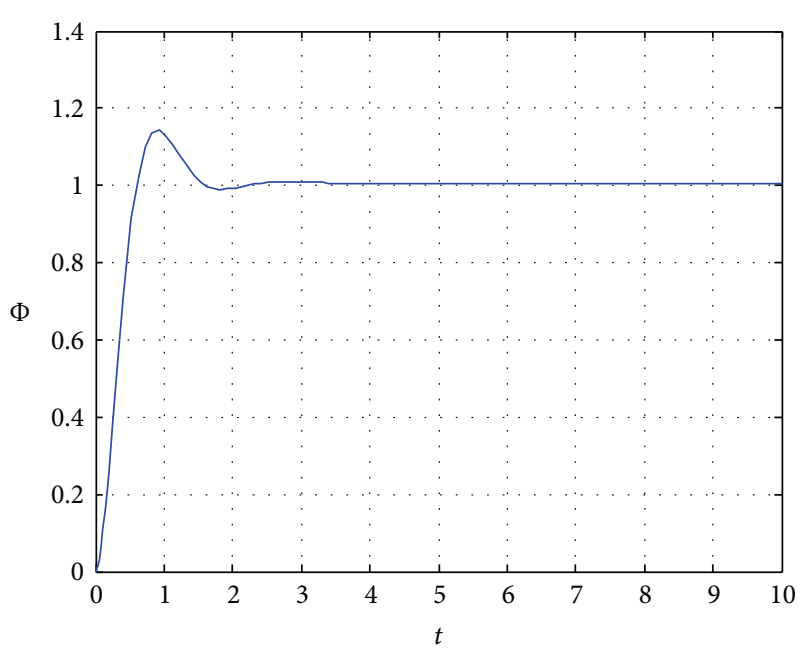

FIGURE 17: Step response on Part 2.

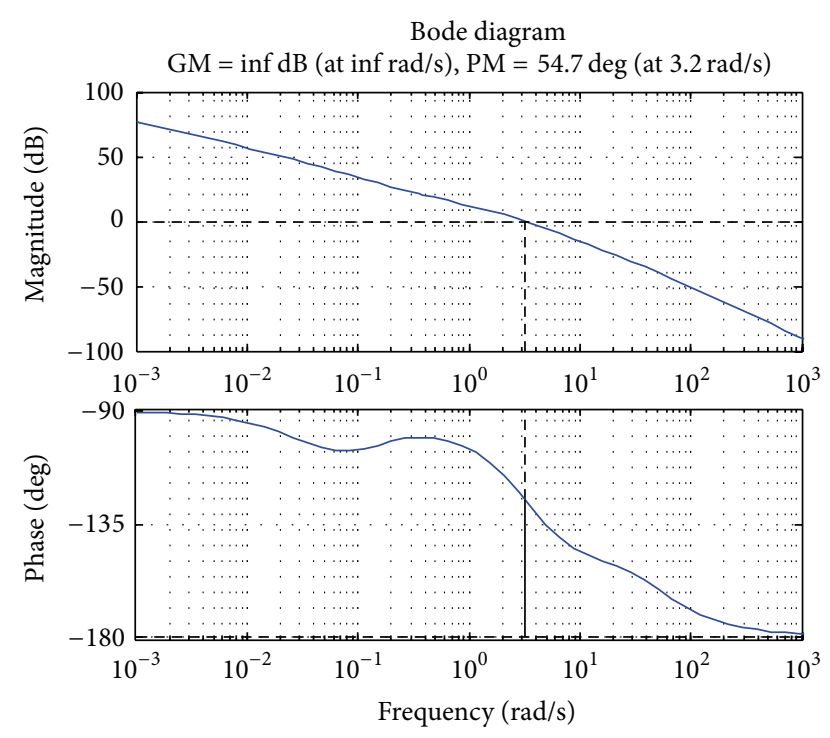

FIgURE 18: Bode diagram on Part 2.

3.0. The step response is shown in Figure 19, of which the rise time is $0.66 \mathrm{~s}$ and the overshoot is $14.6 \%$. The Bode diagram is shown in Figure 20, in which GM is infinity and PM is $54 \mathrm{deg}$. The control system response meets the requirements of control system.

In the region of Part 4 , at the point $(\alpha=12, \mathrm{Ma}=1.1) \epsilon$ $S_{1}$, the control parameter is $K_{p}=9.3, K_{i}=1.9$, and $K_{w}=$ 1.8. The step response is shown in Figure 21, of which the rise time is $0.61 \mathrm{~s}$ and the overshoot is $15.9 \%$. The Bode diagram is shown in Figure 22, in which GM is infinity and PM is $54 \mathrm{deg}$. The PID controller is designed to improve system dynamic performance.

The initial position of the missile is $\left(x_{m}=0, y_{m}=\right.$ $\left.5000 \mathrm{~m}, z_{m}=0\right)$. The position of the target is $\left(x_{t}=0, y_{t}=\right.$ $\left.5000 \mathrm{~m}, z_{t}=0\right)$. The guidance method is proportional navigation. The simulation results are shown in Figure 23.

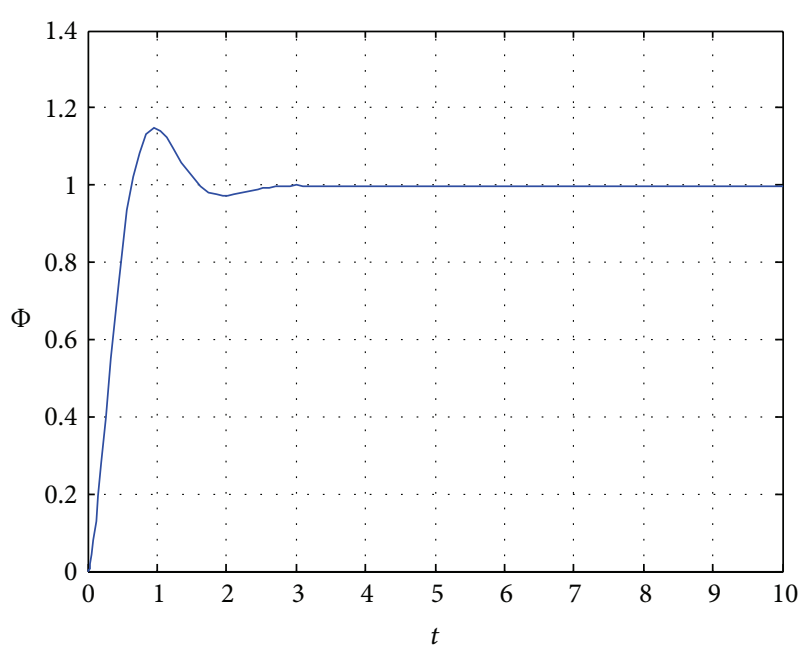

Figure 19: Step response on Part 3.
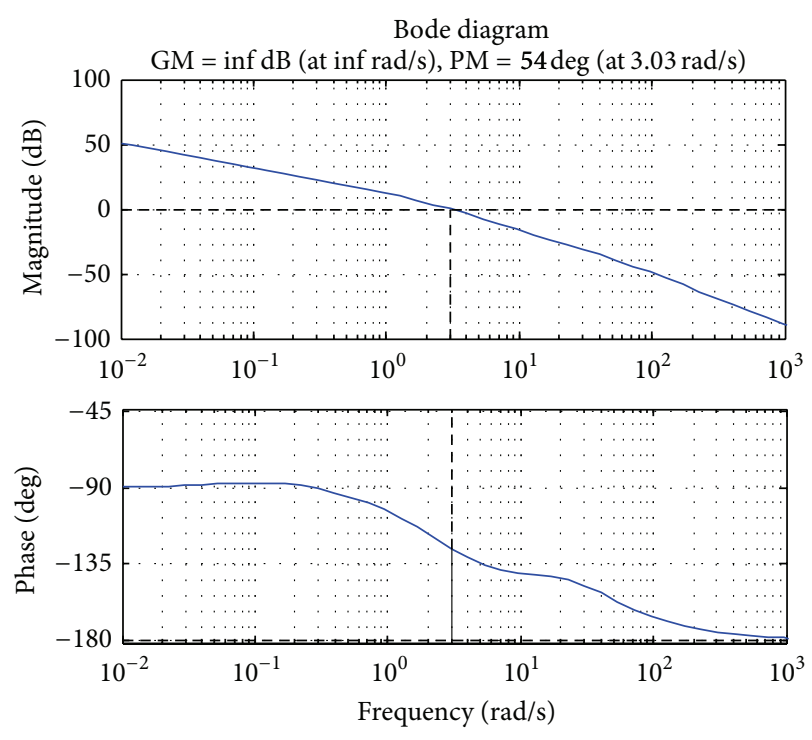

Figure 20: Bode diagram on Part 3.

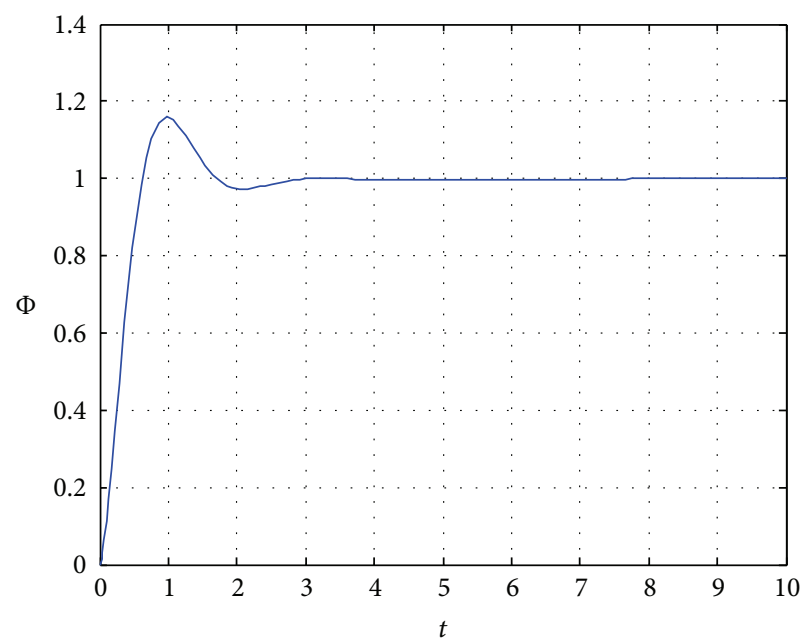

Figure 21: Step response on Part 4. 

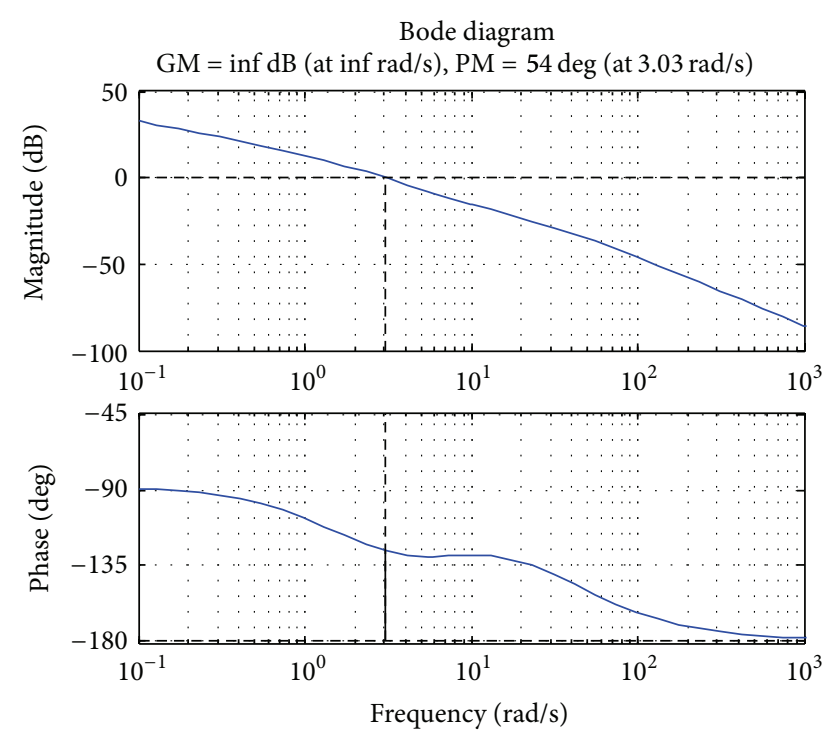

Figure 22: Bode diagram on Part 4.

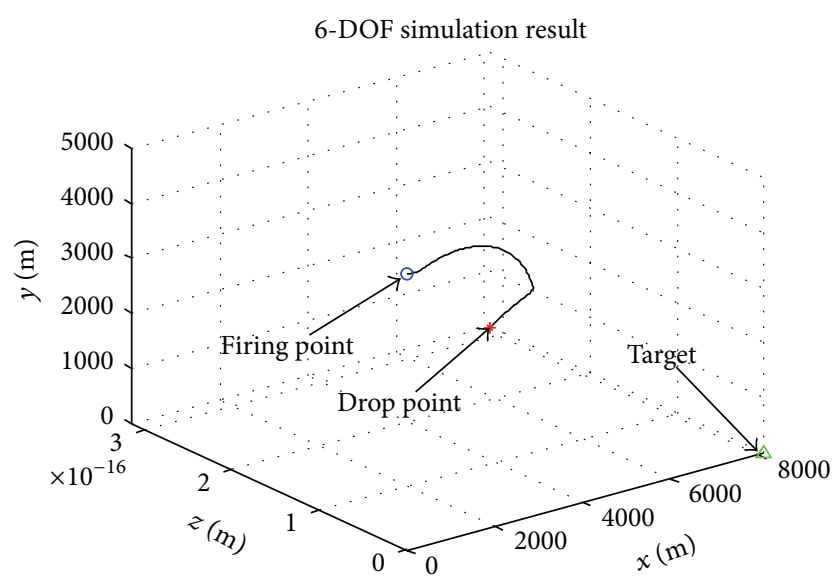

FIGURE 23: 6-DOF simulation.

\section{Conclusion}

In this paper, there are three main research results. First, nonlinearity index algorithms of three models are introduced. The algorithm of nonlinearity index according to engineering application is first proposed. The nonlinearity index can not only qualitatively and quantitatively measure the nonlinear strength of any models but also indicate the source of nonlinearity. The nonlinearity index method is extended to dynamical system. The four nonlinearity index expressions, including static state, static input, dynamic state, and dynamic input nonlinearity indices, are obtained to measure the nonlinear strength of dynamics model. Second, based on nonlinear strength analysis, a new control idea of missile controller design is first proposed. Third, combined with the linear model of missile motion, the simulation results of the nonlinearity indices are given. According to engineering application, the algorithm of nonlinearity index is introduced. Mach number and angle of attack flight envelope are first set as the parameters of the simulation results, and the results show that the nonlinear strength is high at a low Mach number region. Based on the nonlinearity index theory, the missile control system is analyzed. The simulation results indicate that the method discussed in this paper is correct.

The research achievements of this paper can offer some references to the control system design of the strong nonlinear aircraft system. To make the control system have a better stability and robustness, more advanced control algorithm should be considered according to the nonlinear degree of the dynamic model for the future work.

\section{Nomenclature}

९, $\psi, \gamma: \quad$ Missile pitch, yaw, and roll angles

$\theta, \psi_{v}$ : Trajectory inclination, trajectory deflection angles

$I_{x}, I_{y}, I_{z}$ : Moments of inertia about $x, y$, and $z$ body axes

$V: \quad$ Missile total velocity

$m: \quad$ Missile mass

Ma: $\quad$ Mach number

$\alpha, \beta: \quad$ Attack, sideslip angles

$v: \quad$ Nonlinearity index

$\lambda: \quad$ Varying parameter vector

$\Phi: \quad$ Transition matrix.

\section{Conflict of Interests}

The authors declare that there is no conflict of interests regarding the publication of this paper.

\section{References}

[1] P. A. Ioannou and A. Pitsillides, Modeling and Control of Complex Systems, CRC Press, 2007.

[2] S. T. Mohyud-Din, M. A. Noor, and K. I. Noor, "Some relatively new techniques for nonlinear problems," Mathematical Problems in Engineering, vol. 2009, Article ID 234849, 25 pages, 2009.

[3] K. J. Astrom, A. Pedro, B. Mogens, S. Walter, and I. Alberto, Control of Complex Systems, Springer, 2001.

[4] J. Junkins and P. Singla, "How nonlinear is it?: a tutorial on nonlinearity of orbit and attitude dynamics," The Journal of the Astronautical Sciences, vol. 52, no. 1-2, pp. 7-60, 2004.

[5] A. Omran and B. Newman, "Nonlinearity index theory for aircraft dynamic assessment," Journal of Guidance, Control, and Dynamics, vol. 36, no. 1, pp. 293-303, 2013.

[6] K. Zhou, J. Doyle, and K. Glover, Robust and Optimal Control, Prentice Hall, Upper Saddle River, NJ, USA, 1996.

[7] S. Yin, S. X. Ding, X. Xie, and H. Luo, "A review on basic datadriven approaches for industrial process monitoring," IEEE Transactions on Industrial Electronics, vol. 61, no. 11, pp. 64186428, 2014

[8] S. Theodoulis, V. Gassmann, P. Wernert, L. Dritsas, I. Kitsios, and A. Tzes, "Guidance and control design for a class of spin-stabilized fin-controlled projectiles," Journal of Guidance, Control, and Dynamics, vol. 36, no. 2, pp. 517-531, 2013.

[9] J. R. Cloutier and D. T. Stansbery, "Nonlinear, hybrid bankto-turn/skid-to-turn missile autopilot design," in Proceedings of 
the AIAA Guidance, Navigation, and Control Conference and Exhibit, AIAA-2001-4158, August 2001.

[10] R. Mu and X. Zhang, "Control allocation design of reaction control system for reusable launch vehicle," Abstract and Applied Analysis, vol. 2014, Article ID 541627, 13 pages, 2014.

[11] G. M. Siouris, Missile Guidance and Control Systems, Springer, New York, NY, USA, 2004.

[12] D. Lin, J. Fan, Z. Qi, and Y. Mou, "Analysis and improvement of missile three-loop autopilots," Journal of Systems Engineering and Electronics, vol. 20, no. 4, pp. 844-851, 2009.

[13] S. Yin and Z. Huang, "Performance monitoring for vehicle suspension system via fuzzy positivistic C-means clustering based on accelerometer measurements," IEEE/ASME Transactions on Mechatronics, 2014.

[14] D. J. Stilwell and W. J. Rugh, "Interpolation of observer state feedback controllers for gain scheduling," IEEE Transactions on Automatic Control, vol. 44, no. 6, pp. 1225-1229, 1999.

[15] F. Mendez-Vergara, I. Cervantes, and A. Mendoza-Torres, "Stability of gain scheduling control for aircraft with highly nonlinear behavior," Mathematical Problems in Engineering, vol. 2014, Article ID 906367, 12 pages, 2014.

[16] S. Theodoulis and G. Duc, "Missile autopilot design: gainscheduling and the gap metric," Journal of Guidance, Control, and Dynamics, vol. 32, no. 3, pp. 986-996, 2009.

[17] V. Lakshmikantham, X. Liu, and S. Leela, "Variational Lyapunov method and stability theory," Mathematical Problems in Engineering, vol. 3, no. 6, pp. 555-571, 1998.

[18] B. Sasu, "Robust stability and stability radius for variational control systems," Abstract and Applied Analysis, vol. 2008, Article ID 381791, 29 pages, 2008.

[19] A. Dilley, R. M. Bride, V. Yuki, M. Brasher, and E. Marguart, "Dynamic wind tunnel test of an innovative rolling missile model," in Proceedings of the 26th AIAA Aerodynamic Measurement Technology and Ground Testing Conference, 2008.

[20] A. Pankkonen, "Method for rapid modelling of missile aerodynamics," in Proceedings of the AIAA Atmospheric Flight Mechanics Conference, Portland, Ore, USA, August 2011. 


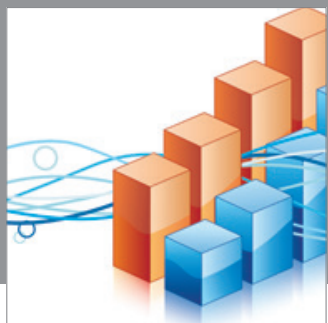

Advances in

Operations Research

mansans

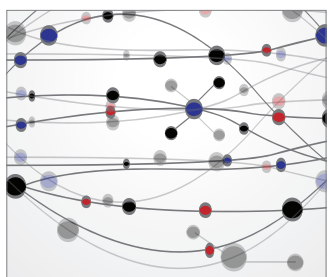

The Scientific World Journal
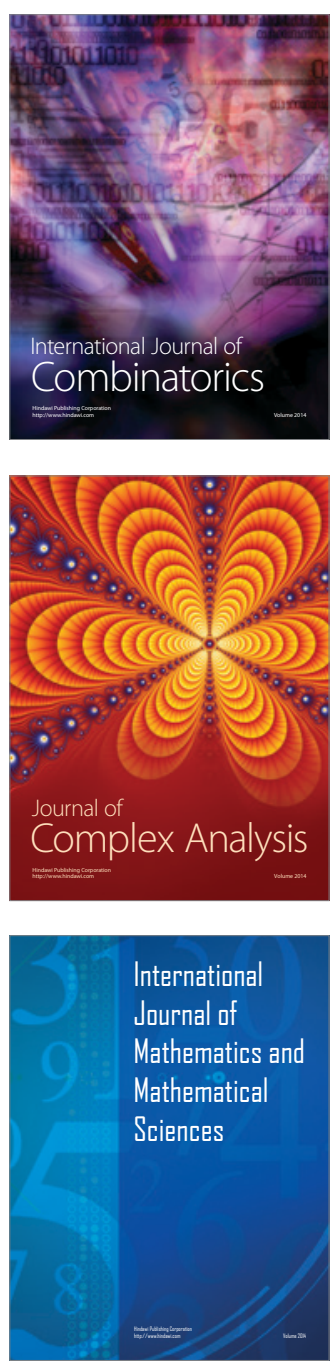
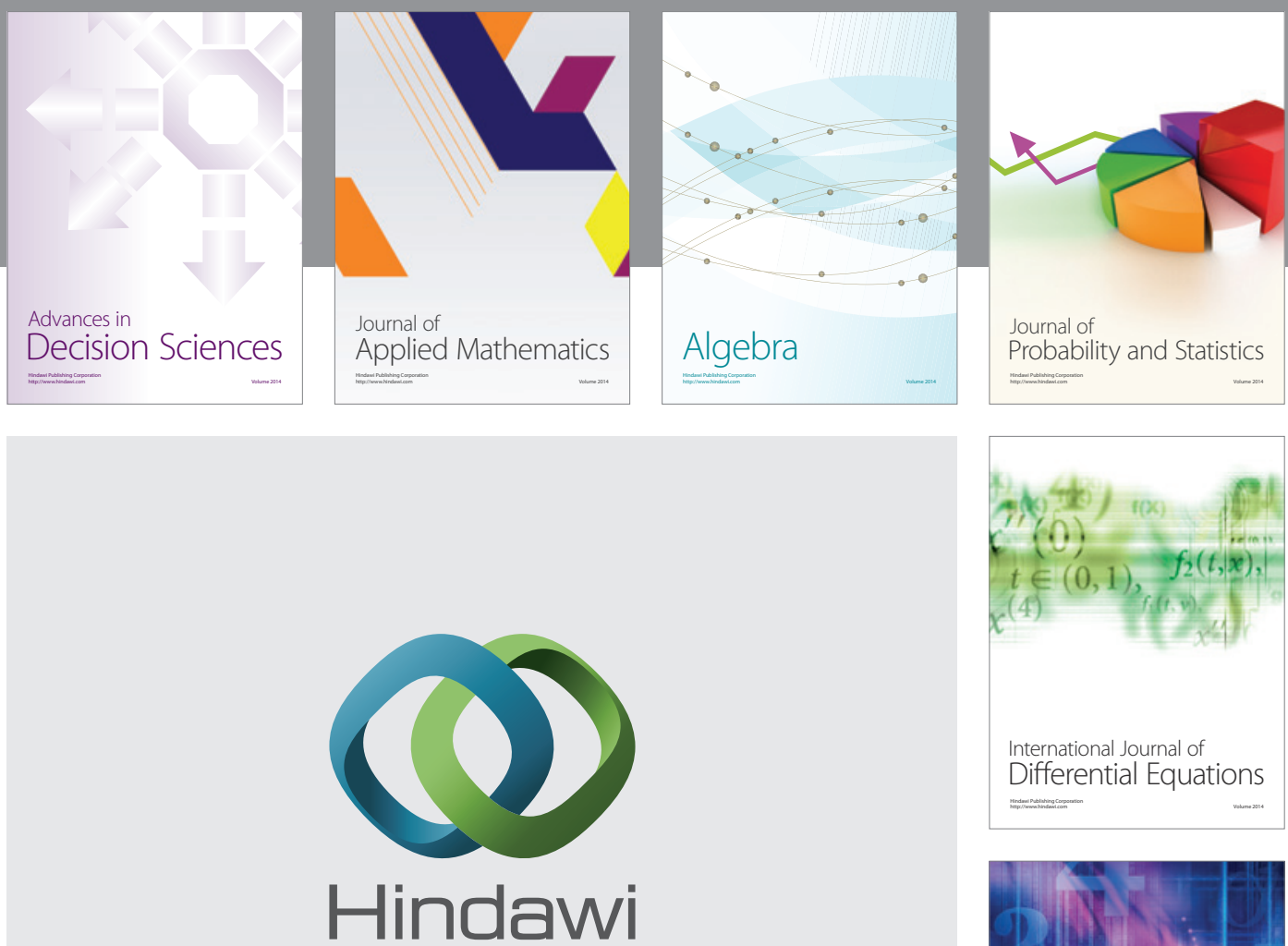

Submit your manuscripts at http://www.hindawi.com
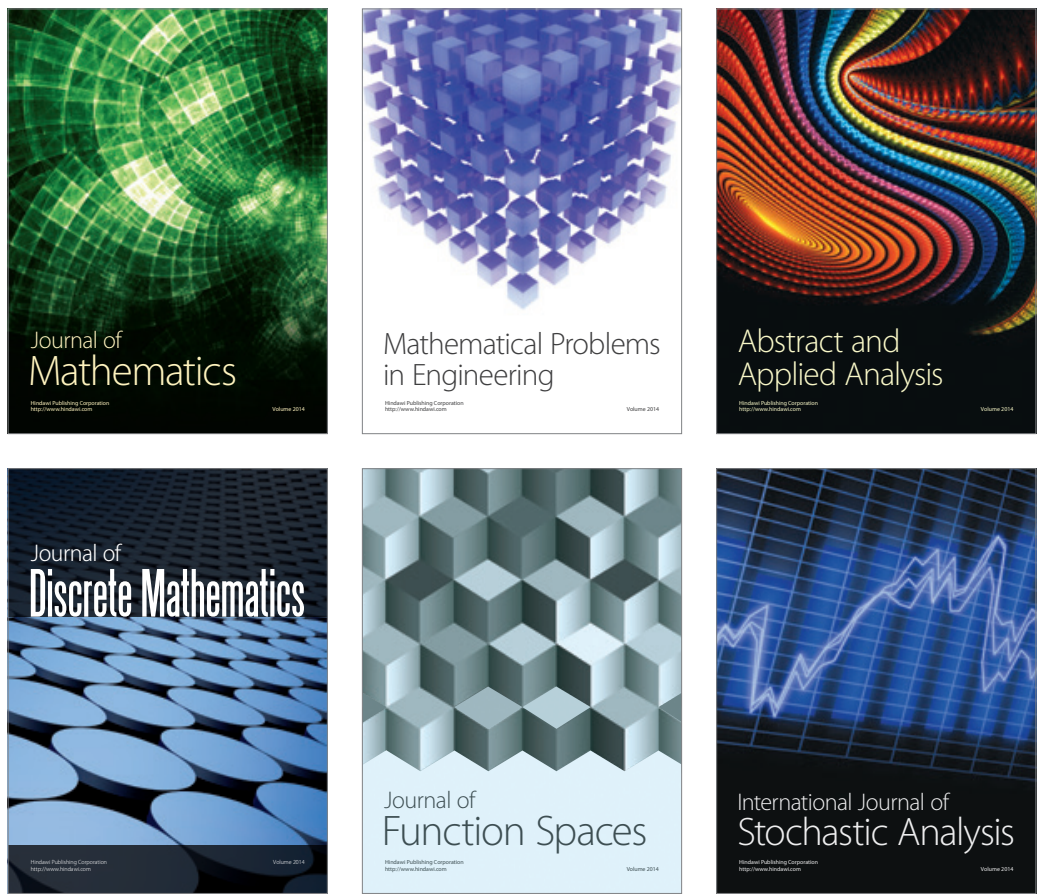

Journal of

Function Spaces

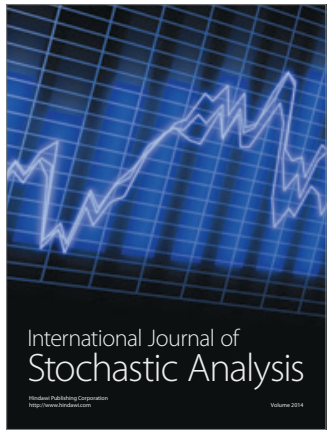

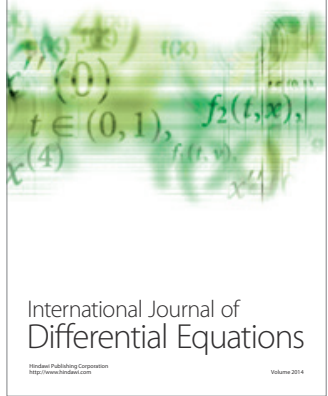
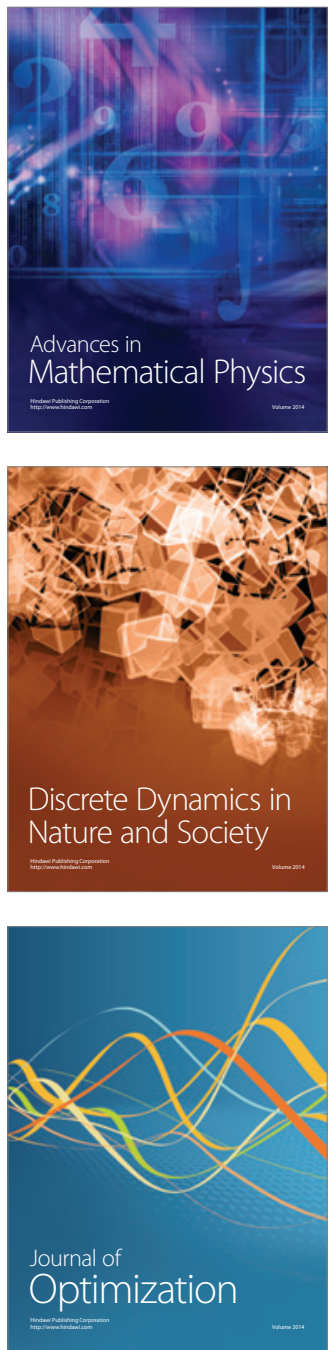